\title{
Formation of the $-\mathrm{N}(\mathrm{NO}) \mathrm{N}(\mathrm{NO})$ - polymer at high pressure and stabilization at ambient conditions
}

\author{
Hai Xiao, Qi An, William A. Goddard III' Wei-Guang Liu, and Sergey V. Zybin \\ Materials and Process Simulation Center, California Institute of Technology, Pasadena, CA 91125 \\ Contributed by William A. Goddard III, December 31, 2012 (sent for review November 12, 2012)
}

\begin{abstract}
A number of exotic structures have been formed through highpressure chemistry, but applications have been hindered by difficulties in recovering the high-pressure phase to ambient conditions (i.e., one atmosphere and $300 \mathrm{~K}$ ). Here we use dispersion-corrected density functional theory [PBE-ulg (Perdew-Burke-Ernzerhof flavor of DFT with the universal low gradient correction for long range London dispersion)] to predict that above 60 gigapascal (GPa) the most stable form of $\mathrm{N}_{2} \mathrm{O}$ (the laughing gas in its molecular form) is a one-dimensional polymer with an all-nitrogen backbone analogous to cis-polyacetylene in which alternate $\mathbf{N}$ are bonded (ionic covalent) to $O$. The analogous trans-polymer is only $0.03 \sim 0.10 \mathrm{eV} /$ molecular unit less stable. Upon relaxation to ambient conditions, both polymers relax below $14 \mathrm{GPa}$ to the same stable nonplanar trans-polymer. The predicted phonon spectrum and dissociation kinetics validates the stability of this trans-poly-NNO at ambient conditions, which has potential applications as a type of conducting nonlinear optical polymer with all-nitrogen chains and as a highenergy oxidizer for rocket propulsion. This work illustrates in silico materials discovery particularly in the realm of extreme conditions (very high pressure or temperature).
\end{abstract}

DFT | high pressure physics and chemistry | prediction of novel materials

$\mathbf{W}$ ith strong interplay between experiment and theory, such molecular crystals as $\mathrm{N}_{2}(1,2), \mathrm{CO}_{2}(3,4), \mathrm{CO}(5,6), \mathrm{NH}_{3}$ (7), and benzene (8) have been transformed into extended solids (covalent and ionic bonded networks) under high pressures. These studies have enhanced our understanding of chemical bonds under compression and provide opportunities to seek additional novel materials; however, it has been difficult to retain these remarkable structures at the ambient conditions needed for most applications (9). For $\mathrm{CO}_{2}$, a 3D covalent network was synthesized (3) at high pressure $(40 \mathrm{GPa})$ and temperature $(1,800 \mathrm{~K})$ that is isomorphic to the $\beta$-cristobalite phase of $\mathrm{SiO}_{2}(10)$, with each carbon atom bonded tetrahedrally to four oxygen atoms. This phase of $\mathrm{CO}_{2}$ was proposed to have potential applications as superhard (initial experiments estimated a bulk modulus of $365 \mathrm{GPa}(11)$, but theory and experiment later found it to be $136 \mathrm{GPa}(10,12))$, nonlinear optical, and high-energy density material, so efforts were made to quench this phase down to $1 \mathrm{~atm}$ and $300 \mathrm{~K}$ (3); however, it reverts back to the molecular phase at pressures lower than $1 \mathrm{GPa}$.

Because it is isoelectronic to $\mathrm{CO}_{2}$ but polar, attempts were made to form an extended solid from $\mathrm{N}_{2} \mathrm{O}$ using compression (above 20 $\mathrm{GPa}$ ) and laser heating (above 1,000 K) in a diamond anvil (13). However, instead it decomposed into a mixture of an ionic crystal $\mathrm{NO}^{+} \mathrm{NO}_{3}{ }^{-}$and compressed $\mathrm{N}_{2}$ molecules. No covalent extended framework similar to the polymeric $\mathrm{CO}_{2}$ phase was found. Indeed, because the nitrogen atom forms one less covalent bond than the carbon atom, it is not obvious that it would be possible to construct a dense extended solid phase of $\mathrm{N}_{2} \mathrm{O}$ other than the ionic form. Even so, we decided to use first principle method to explore a large number of space groups and bonding patterns at high pressures.

Because we start with molecular crystals of nitrous oxide (NNO) in which London dispersion attractions (van der Waals attraction) is dominant, we used the PBE-ulg flavor of density functional theory (DFT-ulg) in which corrections accounting for London dispersion attractions are included (14). We searched for highpressure structures with two independent strategies.

In the first approach, we started with 41 known $\mathrm{AB}_{2}$-type crystal structures, replaced A with $\mathrm{O}$ and $\mathrm{B}$ with $\mathrm{N}$, and used DFT to determine the optimum atom positions and packings using a fixed density of $3.915 \mathrm{gm} / \mathrm{cm}^{3}$ (a relative compression of 0.384 and the same as polymeric $\mathrm{CO}_{2}$ at $41 \mathrm{GPa}$ ) (10). Then we took the new configurations from the lowest energy structures, built appropriate extended cells, and refined further with DFT to obtain the optimum stacking.

In the second approach, we used the USPEX (Universal Structure Predictor: Evolutionary Xtallography) code (15-17) based on an evolutionary algorithm developed by Romanov, Glass, and Lyakhov (15) and featuring local optimization, realspace representation, and flexible physically motivated variation operators. Here we considered a 12 -atom unit cell at 40,60, and $80 \mathrm{GPa}$.

\section{Results}

Both methods led to the same two final high-pressure structures: both corresponding to a $1 \mathrm{D} \mathrm{N}_{2} \mathrm{O}$ polymer but with either $\mathrm{cis}$ - (Fig. $1 B$ ) and trans- (Fig. 1C) conformations (cis is lower), predicted to be the most stable species above $60 \mathrm{GPa}$. The valence bond descriptions of these structures has an alternating $\mathrm{N}$ and $\mathrm{N}^{+}$backbone polyacetylene like zigzag chain with each $\mathrm{N}^{+}$forming a covalent bond to $\mathrm{O}^{-}$. The valence bond description would suggest alternating single and double bonds (1.448 $\AA$ and $1.309 \AA$ ), which we find for cis; however, for trans we find all $\mathrm{NN}$ bonds to have the same bond distances ( $1.390 \AA$ at $1 \mathrm{~atm})$, indicating full resonance along the chain. Fig. 1 shows the enthalpies of various relevant phases of $\mathrm{N}_{2} \mathrm{O}$ as a function of pressure, taking the ground state molecular crystal with space group $\mathrm{Pa} \overline{3}$ ( $\alpha$-nitrogen structure) as the reference. For the two molecular phases, $\mathrm{Pa} \overline{3}$ and space group $\mathrm{Cmca}$, experiments find head-to-tail orientation disorder (18), so we considered all possible conformations in the unit cell to determine the lowest energy. The calculated dependence of crystal volume on pressure is comparable with experiment (Fig. $\mathrm{S} 1$ ), leading to a calculated transition pressure between the two molecular phases of $5.8 \mathrm{GPa}$ at $0 \mathrm{~K}$, compared with the experimental value of $4.8 \mathrm{GPa}$ at room temperature (18).

In the range of $10-55 \mathrm{GPa}$ and $1,000-3,400 \mathrm{~K}$, the diamond anvil experiments on NNO observed a mixture of the ionic $\mathrm{NO}^{+}$ $\mathrm{NO}_{3}{ }^{-}$phase in equilibrium with an $\mathrm{N}_{2}$ molecule phase, formed from the dissociation of $\mathrm{N}_{2} \mathrm{O}$ at high pressure and temperature $(13,19,20)$. We also calculated these phases at the pressures considered here. However, the structure of $\mathrm{NO}^{+} \mathrm{NO}_{3}^{-}$is not well-established experimentally: experiments reported the formation of monoclinic $P 2_{1} / m$ phase ionic salt from the high-pressure

Author contributions: H.X., W.A.G., and S.V.Z. designed research; H.X., Q.A., W.A.G., and W.-G.L. performed research; H.X., Q.A., W.A.G., W.-G.L., and S.V.Z. analyzed data; and H.X., Q.A., W.A.G., W.-G.L., and S.V.Z. wrote the paper.

The authors declare no conflict of interest.

${ }^{1}$ To whom correspondence should be addressed. E-mail: wag@wag.caltech.edu.

This article contains supporting information online at www.pnas.org/lookup/suppl/doi:10. 1073/pnas.1222890110/-/DCSupplemental. 
B
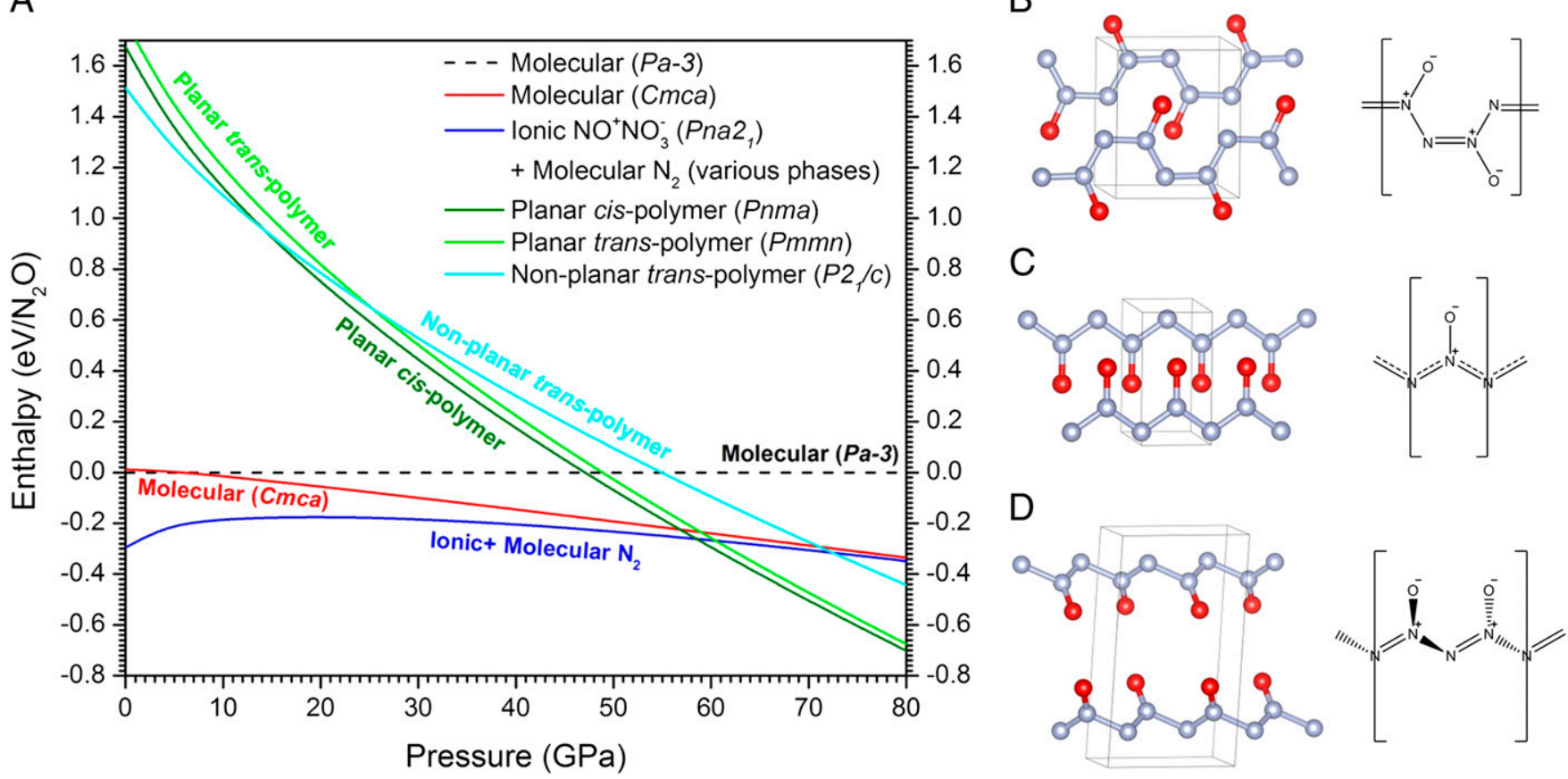

Fig. 1. (A) Ground state enthalpy as a function of pressure predicted for various phases of $\mathrm{N}_{2} \mathrm{O}$, with illustrations of crystal structures and chemical formulations of single chain for planar cis-polymer $(B)$, planar trans-polymer $(C)$, and nonplanar trans-polymer $(D)$.

(2 GPa) reaction of an $\mathrm{N}_{2}$ and $\mathrm{O}_{2}$ mixture (21), whereas the original experiments on dissociation of $\mathrm{N}_{2} \mathrm{O}$ under laser heating in a diamond anvil found an orthorhombic phase related to the aragonite to form after cooling $(13,22)$. Therefore, for the DFT study, we constructed the $\mathrm{NO}^{+} \mathrm{NO}_{3}{ }^{-}$crystal by cell relaxation starting with the aragonite structure. This led to an orthorhombic $\mathrm{Pna}_{1}$ phase (Fig. S2 and Table S1), which we found to be $0.03 \mathrm{eV}$ per formula more stable than the monoclinic phase. We carried out DFT calculations of $\mathrm{N}_{2}$ molecular crystals, starting with the known phases $(\alpha, \gamma, \varepsilon, \zeta)$ at low temperature (23), and at each pressure, the energy calculated to be most stable was used in the enthalpy calculations. Surprisingly, this combination of $\mathrm{NO}^{+} \mathrm{NO}_{3}{ }^{-}$ and $\mathrm{N}_{2}$ is more stable than the NNO molecular phases for all pressures down to $0 \mathrm{GPa}$. This is plausible, as the heat of formation of $\mathrm{N}_{2} \mathrm{O}$ molecule is $82 \mathrm{~kJ} / \mathrm{mol}(1 \mathrm{kcal}=4.18 \mathrm{~kJ})$ above $\mathrm{N}_{2}$ and $\mathrm{O}_{2}$, which were shown to form $\mathrm{NO}^{+} \mathrm{NO}_{3}{ }^{-}$at $2 \mathrm{GPa}$ using $10.2 \mathrm{keV}$ synchrotron $\mathrm{X}$-ray radiation (21).

Fig. 1 shows that the cis and trans $1 \mathrm{D}$ polymeric phases remain energetically close ( is more stable by $0.03 \sim 0.10 \mathrm{eV} /$ molecular unit) and become more stable than both the molecular and the mixed ionic phases at pressures above $60 \mathrm{GPa}$. Including zero point energy (ZPE) and entropy from phonons has little effect (Fig. S3):

- including ZPE to obtain the $0 \mathrm{~K}$ equation of state (EOS), we find that the critical pressure for the transition from the mixed ionic phase to the cis-polymer increases from $58.5 \mathrm{GPa}$ to $61 \mathrm{GPa}$,

- while introducing entropy and enthalpy corrections to $300 \mathrm{~K}$ leads to a further increase to $62 \mathrm{GPa}$ at $300 \mathrm{~K}(64 \mathrm{GPa}$ at $500 \mathrm{~K})$.

The dominant factor in determining the enthalpy at high pressure is the compressibility. For the mixed phase, the ionic $\mathrm{NO}^{+} \mathrm{NO}_{3}{ }^{-}$ component is a dense solid, but the global compressibility of the mixed phase is dominated by the molecular phase of $\mathrm{N}_{2}$, which polymerizes only above $110 \mathrm{GPa}(2)$. In contrast, the two new NNO polymeric phases each forms a pure extended solid with mixed covalent and ionic frameworks. Thus, these phases dominate at high pressures (where the PV term in the enthalpy becomes dominant) over all of the other phases of $\mathrm{N}_{2} \mathrm{O}$ containing molecular forms.

The major difference between these cis and trans forms is that the planar cis-NNO polymer possesses alternating single and double bonds as in polyenes (polyacetylene) with an even number of carbons, whereas the planar trans-conformation of NNO has equal NN bond lengths (strong resonance) along the chain, perhaps due to strong electrostatic repulsion between adjacent negatively charged oxygen atoms.

We further investigated the stability of the two NNO polymers by phonon analysis. Indeed, for both crystals we find no imaginary phonon modes under high pressures, as shown in the phonon spectra in Fig. 2 (at $80 \mathrm{GPa}$ ), which confirms their existence as stable species (local minima). However, as the pressure is released, both crystals develop imaginary phonon modes at $\sim 14$ $\mathrm{GPa}$, and both relax to one single nonplanar trans-conformation (Dataset S1) at zero pressure, with a phonon spectrum that attests its stability. Under high pressures, the planar conformations with their higher compressibilities are more stable, but they lead to larger electrostatic repulsion between negatively charged oxygen atom and lone-pair on nitrogen in the cis-case and between adjacent negatively charged oxygen atoms in the trans-case. It is the competition between compressibility and electrostatics that leads to the transition to the nonplanar polymer at low pressure.

\section{Discussion}

Besides accommodating electrostatics, the transition between planar and nonplanar trans-conformations is also accompanied by an abrupt change from uniform bonding to alternating single and double bonds, as shown in Fig. 3, implying synergistic Peierls distortion, which is relevant to the electronic properties. Therefore, we calculated band gaps of all polymeric structures (optimized with PBE-ulg flavor of DFT) and plotted against pressures in Fig. 3. Interestingly, PBE-ulg predicts that both planar NNO polymers are $1 \mathrm{D}$ metals, with transitions to insulators below 72 $\mathrm{GPa}$ for cis-polymer and $25 \mathrm{GPa}$ for trans-polymer, the latter with 


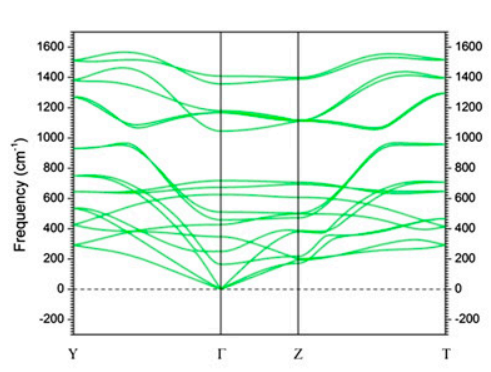

Planar trans-polymer at $80 \mathrm{GPa}$

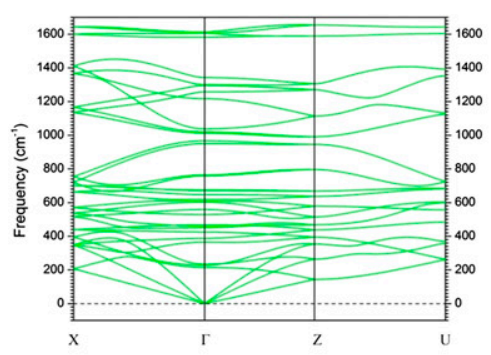

Planar cis-polymer at $80 \mathrm{GPa}$

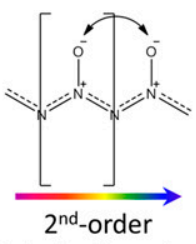

Peierls distortion

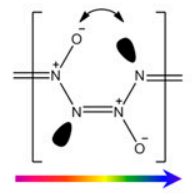

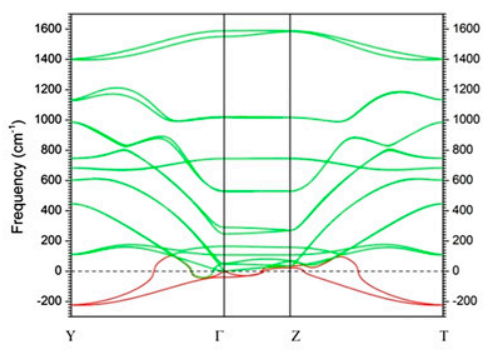

Planar trans-polymer at $\mathbf{0} \mathbf{~ G P a}$

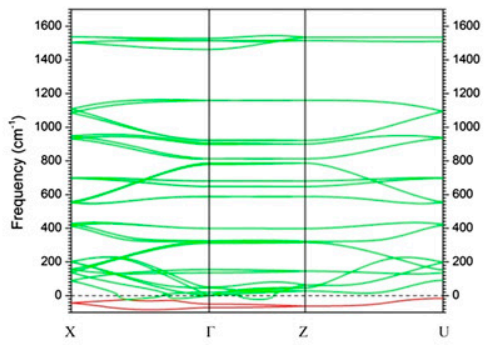

Planar cis-polymer at $\mathbf{0} \mathbf{~ G P a}$

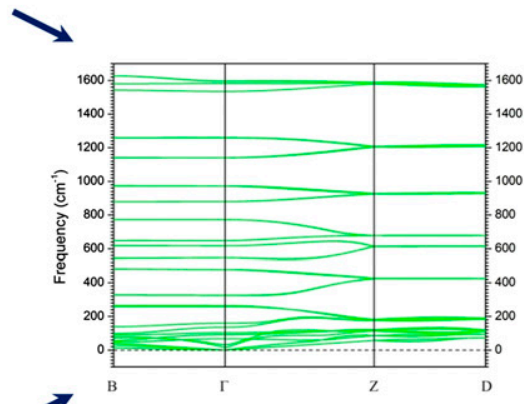

Non-planar trans-polymer at $\mathbf{0} \mathbf{~ G P a}$

Fig. 2. Phonon spectra for polymer phases of $\mathrm{N}_{2} \mathrm{O}$ predicted at 80 and $0 \mathrm{GPa}$. Both planar cis- and trans-polymer phases develop imaginary phonon modes, due to electrostatic repulsions and/or second-order Peierls distortion, and transform into the same nonplanar trans-polymer phase, the phonon spectrum of which at $0 \mathrm{GPa}$ shows no imaginary modes.

simultaneous conformation transition from planar to nonplanar. Fig. 3 also shows the bond length differences between neighboring $\mathrm{N}-\mathrm{N}$ bonds for the various phases. We find a trend from uniform bonding (exact for trans-polymer, quasi-uniform for cis-polymer with $0.03 \AA$ difference) at high pressures to alternating single and double bonds when pressure is released. Combining this with the trend in band gaps indicates a first-order Peierls distortion as in polyacetylene. However, it is well documented $(24,25)$ that density functionals, such as PBE that are based only on local density approximation and generalized gradient approximation, significantly underestimate band gaps for insulators, due to intrinsic delocalization error (26) from including the self-interaction in the
Coulomb energy. To verify the metallic properties of these polymers under high pressures, we reinvestigated all electronic structures with the hybrid functional B3PW91 (27), which we showed to predict accurate band gaps for a wide range of semiconductors (28). In contrast to PBE, B3PW91 predicts insulating states for all three polymers throughout the whole range of pressures considered, as shown in Fig. 3. Here the density of states (Fig. S4) shows that both planar cis- and trans-polymers at high pressures with (quasi-) uniform bonding are charge-transfer insulators, with the valence band dominated by negatively charged oxygen and bridging nitrogen atoms, whereas the conduction band is dominated by the positively charged nitrogen atom. Accordingly, B3PW91 results
A

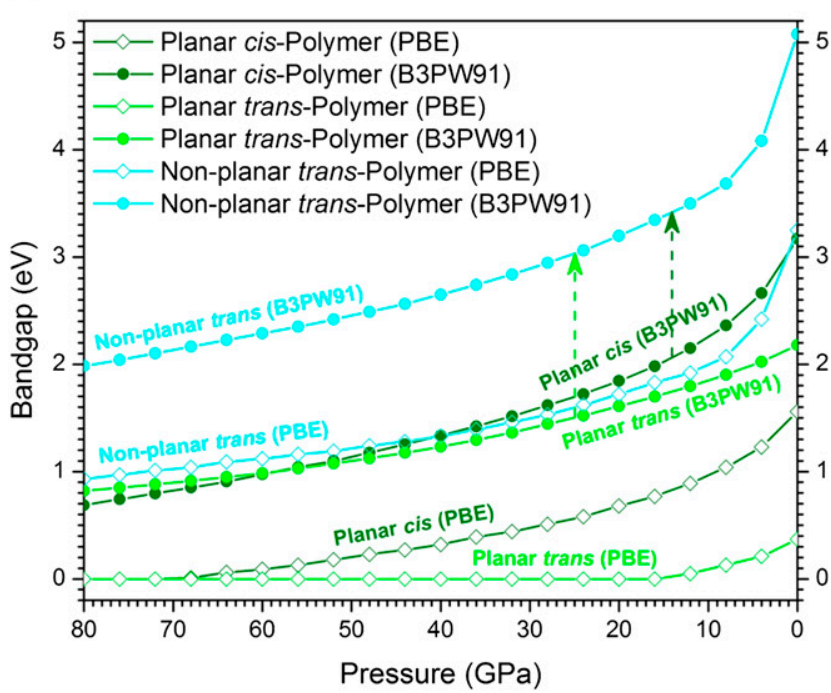

B

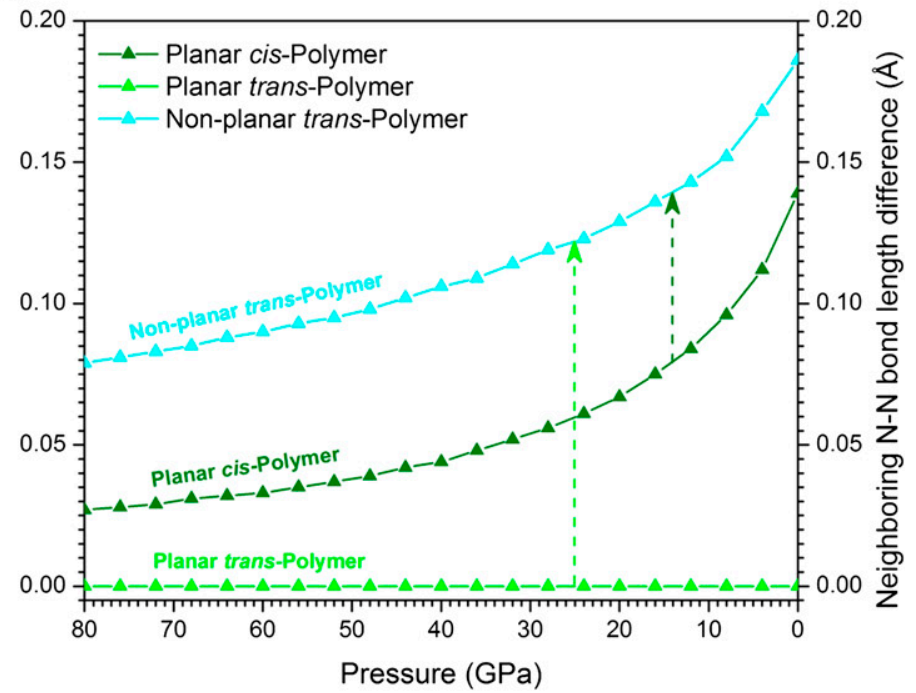

Fig. 3. Band gap $(A)$ and neighboring $N-\mathrm{N}$ bond length difference $(B)$ predicted as functions of pressure for all three polymer phases of $\mathrm{N}_{2} \mathrm{O}$ at ground states. The dashed lines with arrows indicate where the transition from planar to nonplanar conformation happens. 
infer the transition to be second-order Peierls distortion as in polyacene. At this point we cannot be sure whether to trust the B3PW91 or the PBE description, so it would be most valuable to carry out experiments on the conductivity or electronic spectra of these new phases.

To further evaluate the stability of nonplanar trans-polymer, we investigated the dissociation kinetics of the polymer, using DFT for a model oligomer containing $8 \mathrm{~N}_{2} \mathrm{O}$ units and terminated with methyl groups at both ends. We stretched this finite chain until it fractured, locating the transition state (TS) shown in Fig. 4. This leads to an activation energy of $20.6 \mathrm{kcal} / \mathrm{mol}$. At the TS, the partially dissociated $\mathrm{N}_{2} \mathrm{O}$ has $N$-N distances of 2.062 and $1.830 \AA$ to its two neighbors, much longer than corresponding equilibrium distances of 1.465 and $1.447 \AA$. This indicates that a large free space is required to activate the dissociation process, making the dissociation less favorable in the solid. This highactivation barrier of $20.6 \mathrm{kcal} / \mathrm{mol}$ from the finite model corresponds to a surface energy of $1.56 \times 10^{3} \mathrm{erg} / \mathrm{cm}^{2}$, considering the packing in the crystal. Thus, we consider that it is likely that the nonplanar trans-polymer of $\mathrm{N}_{2} \mathrm{O}$ will be stable at $1 \mathrm{~atm}$ pressure and $300 \mathrm{~K}$.

After passing the TS, the oligomer would start releasing $\mathrm{N}_{2} \mathrm{O}$ one by one from each end, accompanied with significant heat release of $40.6 \mathrm{kcal} / \mathrm{mol}$ per $\mathrm{N}_{2} \mathrm{O}$ molecule, which might lead to catastrophic decomposition. Thus, the nonplanar trans-NNO polymer is a high-energy content structural material. Indeed, we calculated (PBE-ulg) the nonplanar $\mathrm{N}_{2} \mathrm{O}$ trans-polymer to provide an internal energy release of $3.5 \mathrm{~kJ} / \mathrm{g}$ when dissociated into $\mathrm{N}_{2}$ and $\mathrm{O}_{2}$, which is comparable to the energy release of trinitrotoluene (TNT) $(4.2 \mathrm{~kJ} / \mathrm{g})$. Thus, nonplanar trans-NNO is a potential high-energy oxidizer for new explosive composites and rocket propellants.

Since the $\mathrm{N}_{2} \mathrm{O}$ polymer chain is composed of alternating single and double bonds, similar to polyacetylene, albeit with an all nitrogen backbone, it might form the basis for a unique type of conducting polymer, through appropriate doping or structure modification. Similarly we expect that it might have strong nonlinear polarizabilities for nonlinear optical applications.
Summarizing, we used DFT to predict that the NNO molecular crystal can be transformed into novel polymeric phases at high pressures (beyond decomposition into mixture phase of ionic compound $\mathrm{NO}^{+} \mathrm{NO}_{3}{ }^{-}$and $\mathrm{N}_{2}$ gas previously observed in experiments). The two most stable $1 \mathrm{D} \mathrm{N}_{2} \mathrm{O}$ polymers with planar cis- and transconformations were identified to be energetically favorable at pressures above $60 \mathrm{GPa}$. More importantly, when the pressure is released, these polymers transform into the same nonplanar transconformation, stable at ambient pressure and temperature. This was substantiated by analysis of the phonon spectrum and by calculating the dissociation kinetics. This unique poly-NNO material might be an excellent high-energy oxidizer for a polymer composite in which nonpolar NNO forms the matrix.

The PBE calculations suggest metallic property in the highpressure polymer resulting from the strong resonance in these systems, which seems to disappear in the low-pressure nonplanar polymer due to changes in bonding, leading to Peierls distortion, together with electrostatic repulsions. Thus, with doping these NNO polymers, such as I, which works successfully in polyacetylene, may give rise to a new type of conducting polymer based on all-nitrogen chains. This whole work serves to illustrate the unique in silico process of discovering materials by theoretical modeling particularly under extreme conditions.

\section{Methods}

PBE-ulg calculations were performed using Vienna Ab-initio Simulation Package (VASP) package (29-31), modified to describe PBE-ulg and using the projector augmented wave method (32) to account for core-valence interactions. The kinetic energy cutoff for plane wave expansions was set to 500 $\mathrm{eV}$, and the reciprocal space was sampled by $\Gamma$-centered Monkhorst-Pack scheme with a fine resolution of $2 \pi \times 1 / 60 \AA^{-1}$.

In the search of structures using USPEX (15-17), the kinetic energy cutoff was lowered to $400 \mathrm{eV}$, with reciprocal grids of $2 \pi \times 0.06 \AA^{-1}$. The convergence criteria were set to $1 \times 10^{-6} \mathrm{eV}$ energy difference for solving for the electronic wavefunction and $1 \times 10^{-3} \mathrm{eV} / \AA ̊$ force for geometry optimization.

To obtain the phonon spectra, very tight convergence criteria were used, with $1 \times 10^{-8} \mathrm{eV}$ energy difference and $1 \times 10^{-6} \mathrm{eV} / \AA ̊ \AA$ force thresholds. To calculate force constants, we used the supercell approach with finite displacements, as implemented in the Phonopy code (33).

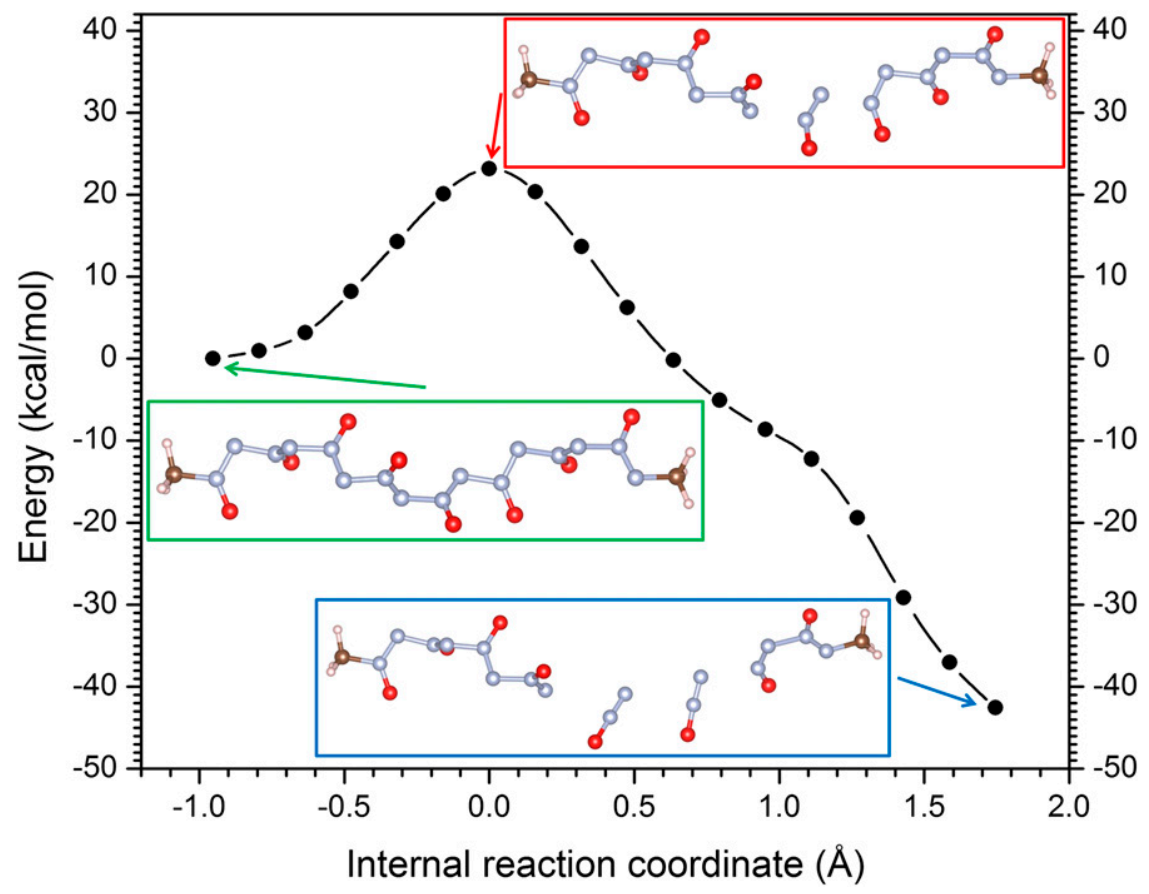

Fig. 4. Dissociation path calculated for dissociation of the model 8-mer. The oligomer contains $8 \mathrm{~N}_{2} \mathrm{O}$ units and is terminated with methyl groups at both ends. 
B3PW91 calculations were conducted using CRYSTAL09 package (34) and $6-311 G *$ triple-zeta quality basis sets for both nitrogen and oxygen. Similar $k$-space grids and convergence criteria as in fine calculations with VASP were set for consistency.

For finite model calculations, the geometry optimization, Hessian calculation, and TS search were carried out at UB3LYP/6-311G*+ level using Jaguar (35). The Hessian was used to provide the vibrational frequencies for ZPE and

1. Mailhiot C, Yang LH, McMahan AK (1992) Polymeric nitrogen. Phys Rev B Condens Matter 46(22):14419-14435.

2. Eremets MI, Gavriliuk AG, Trojan IA, Dzivenko DA, Boehler R (2004) Single-bonded cubic form of nitrogen. Nat Mater 3(8):558-563.

3. lota V, Yoo CS, Cynn H (1999) Quartzlike carbon dioxide: An optically nonlinear extended solid at high pressures and temperatures. Science 283(5407):1510-1513.

4. Serra S, Cavazzoni C, Chiarotti GL, Scandolo S, Tosatti E (1999) Pressure-induced solid carbonates from molecular $\mathrm{CO}_{2}$ by computer simulation. Science 284(5415):788-790.

5. Bernard S, Chiarotti GL, Scandolo S, Tosatti E (1998) Decomposition and polymerization of solid carbon monoxide under pressure. Phys Rev Lett 81(10):2092-2095.

6. Lipp MJ, Evans WJ, Baer BJ, Yoo CS (2005) High-energy-density extended CO solid. Nat Mater 4(3):211-215.

7. Pickard CJ, Needs RJ (2008) Highly compressed ammonia forms an ionic crystal. Nat Mater 7(10):775-779.

8. Ciabini L, et al. (2007) Triggering dynamics of the high-pressure benzene amorph ization. Nat Mater 6(1):39-43.

9. McMillan PF (2002) New materials from high-pressure experiments. Nat Mater 1(1): $19-25$

10. Datchi F, Mallick B, Salamat A, Ninet S (2012) Structure of polymeric carbon dioxide $\mathrm{CO}_{2}$-V. Phys Rev Lett 108(12):125701.

11. Yoo CS, et al. (1999) Crystal structure of carbon dioxide at high pressure: "Superhard" polymeric carbon dioxide. Phys Rev Lett 83(26):5527-5530.

12. Dong JJ, et al. (2000) Investigation of hardness in tetrahedrally bonded nonmolecular $\mathrm{CO}_{2}$ solids by density-functional theory. Phys Rev B 62(22):14685-14689.

13. Somayazulu $\mathrm{M}$, et al. (2001) Novel broken symmetry phase from $\mathrm{N}_{2}$ ) $\mathrm{O}$ at high pressures and high temperatures. Phys Rev Lett 87(13):135504.

14. Kim H, Choi JM, Goddard WA (2012) Universal correction of density functional theory to include London dispersion (up to Lr, element 103). J Phys Chem Lett 3(3):360-363.

15. Oganov AR, Glass CW (2006) Crystal structure prediction using ab initio evolutionary techniques: Principles and applications. J Chem Phys 124(24):244704.

16. Glass CW, Oganov AR, Hansen N (2006) USPEX-Evolutionary crystal structure prediction. Comput Phys Commun 175(11-12):713-720.

17. Lyakhov $A O$, Oganov $A R$, Valle $M(2010)$ How to predict very large and complex crystal structures. Comput Phys Commun 181(9):1623-1632.

18. Mills RL, Olinger B, Cromer DT, Lesar R (1991) Crystal-structures of $\mathrm{N}_{2} \mathrm{O}$ to $12 \mathrm{GPa}$ by X-ray-diffraction. J Chem Phys 95(7):5392-5398.

19. Yoo CS, et al. (2003) Disproportionation and other transformations of $\mathrm{N}_{2} \mathrm{O}$ at high pressures and temperatures to lower energy, denser phases. J Phys Chem B 107(24):5922-5925. thermo-corrections to enthalpy. The TS was shown to have exactly one imaginary vibrational mode by following the minimum energy path scan to connect reactant and product. Enthalpies are reported at $298.15 \mathrm{~K}$ and $1 \mathrm{~atm}$.

ACKNOWLEDGMENTS. This work was supported by the Office of Naval Research (N00014-12-1-0538; program manager, Cliff Bedford) and the Defense Advanced Research Planning Agency (program manager, Judah Goldwasser).

20. lota V, Park JH, Yoo CS (2004) Phase diagram of nitrous oxide: Analogy with carbon dioxide. Phys Rev B 69(6):064106.

21. Meng $\mathrm{Y}$, et al. (2006) Hard x-ray radiation induced dissociation of $\mathrm{N}_{2}$ and $\mathrm{O}_{2}$ molecules and the formation of ionic nitrogen oxide phases under pressure. Phys $\operatorname{Rev} B$ 74(21): 214107.

22. Song Y, Somayazulu M, Mao HK, Hemley RJ, Herschbach DR (2003) High-pressure structure and equation of state study of nitrosonium nitrate from synchrotron $\mathrm{x}$-ray diffraction. J Chem Phys 118(18):8350-8356.

23. Katzke H, Toledano P (2008) Theoretical description of pressure- and temperatureinduced structural phase transition mechanisms of nitrogen. Phys Rev B 78(6):064103.

24. Perdew JP, Levy M (1983) Physical content of the exact Kohn-Sham orbital energies: Band-gaps and derivative discontinuities. Phys Rev Lett 51(20):1884-1887.

25. Sham LJ, Schluter M (1983) Density-functional theory of the energy gap. Phys Rev Lett 51(20):1888-1891.

26. Mori-Sánchez P, Cohen AJ, Yang WT (2008) Localization and delocalization errors in density functional theory and implications for band-gap prediction. Phys Rev Lett 100(14):146401.

27. Becke AD (1993) Density-functional thermochemistry. III. The role of exact exchange. J Chem Phys 98(7):5648-5652.

28. Xiao H, Tahir-Kheli J, Goddard WA (2011) Accurate band gaps for semiconductors from density functional theory. J Phys Chem Lett 2(3):212-217.

29. Kresse G, Hafner J (1993) Ab initio molecular dynamics for liquid metals. Phys Rev $B$ Condens Matter 47(1):558-561.

30. Kresse G, Furthmuller J (1996) Efficiency of ab-initio total energy calculations for metals and semiconductors using a plane-wave basis set. Comput Mater Sci 6(1): 15-50.

31. Kresse G, Furthmüller J (1996) Efficient iterative schemes for ab initio total-energy calculations using a plane-wave basis set. Phys Rev B Condens Matter 54(16): 11169-11186.

32. Kresse G, Joubert D (1999) From ultrasoft pseudopotentials to the projector augmented-wave method. Phys Rev B 59(3):1758-1775.

33. Togo A, Oba F, Tanaka I (2008) First-principles calculations of the ferroelastic transition between rutile-type and $\mathrm{CaCl}_{2}$-type $\mathrm{SiO}_{2}$ at high pressures. Phys $\operatorname{Rev} B$ 78(13): 134106.

34. Dovesi R, et al. (2009) CRYSTAL 2009 User's Manual (Univ of Torino, Torino, Italy). 35. Schrödinger, LLC (2010) Jaguar (Schrödinger, New York), Version 7.7. 